\title{
Broad Fuzzy Neural Control Using Impedance Learning
}

\author{
Haohui Huang ${ }^{1}$, Chenguang Yang ${ }^{*, 2}$, Zhaojie $\mathrm{Ju}^{3}$, Yuxia Yuan ${ }^{1}$ and Zhijun $\mathrm{Li}^{4}$
}

\begin{abstract}
This work proposes a novel control strategy based on broad fuzzy neural network (BFNN) by using impedance learning, which is subjected to contact with the unknown dynamic environment. Compared with the original fuzzy neural network, this framework is provided the prominent feature by taking the advantage of broad learning system (BLS) to approximate the unknown dynamic model. Aiming at offering a compliance contact scheme, this paper introduce the impedance learning to establish the robot-environment interaction model. Also, a stable controller, which is able to tackle the problems related to the state constrain, is designed through Barrier Lyapunov Function (BLF). The proposed method can achieve the favourable tracking action while guaranteeing the stability of closed-loop system. In the end, simulation study is performed to verify the effectiveness of BFNN with a two-DOF manipulator.
\end{abstract}

\section{INTRODUCTION}

In the last few years, increasing robotic manipulator techniques have been adopted into our daily life and industrial appliances. One of the most difficulties in robotics researches is how to achieve the desirable and friendly interaction control in its work-piece. Aiming to enable robot response like a second order mass-spring-damper, it is important for robotic system to have a capability to control not only in motion and position but also in force aspect. The current trend of control schemes in human-machine interaction control are force control [1] and impedance control [2-3] which can offer a desired dynamic motor and force control behavior by regulating the impedance parameters. Several researches have been done on this field. In order to tackle the force tracking problem, Cheah et al. [4] has proposed an iterative impedance learning scheme to approximate the target impedance model by taking actions repeatedly for robotic manipulators. In [5], an adaptive model based impedance controller is designed to achieve the desire physical interaction for human and robot. Li et al. [6] proposed a learning procedure without any identified model to achieve the manipulator compliant interaction behavior. The aforementioned works can lead to stable and desired tracking performance, but the robotic dynamics is indispensable for these algorithms.

This work was partially supported by National Nature Science Foundation (NSFC) under Grants 61861136009 and 61811530281.

${ }^{1} \mathrm{H}$. Huang and Y. Yuan are with the Key Laboratory of Autonomous Systems and Networked Control, College of Automation Science and Engineering, South China University of Technology, Guangzhou 510640, China.

${ }^{2}$ C. Yang is with the Bristol Robotics Laboratory, University of the West of England, Bristol, BS16 1QY, UK.

${ }^{3} \mathrm{Z}$. Ju is with the School of Computing, the University of Portsmouth, Portsmouth PO1 3HE, UK

${ }^{4} \mathrm{Z}$. Li is with the Department of Automation, University of Science and Technology of China, Hefei 230026, China.

*Corresponding author. Email: cyang@iee.org.
In the field of impedance controller design for humanmachine interaction, uncertainties and unknown disturbance is the crucial problems for the robotics system. Recent researches have proven that adaptive Neural Networks(NN) and fuzzy systems have a prominent learning capability to approximate any nonlinear and continous functions to any specific accuracy [7]-[9]. In [10], an NN impedance control is designed with the presence of uncertainties by considering a target impedance reference trajectory. Ping et al. [11] designed an impedance control law by using the adaptive fuzzy approximators to improve the interacted behavior for the robot with the uncertain dynamics. However, fuzzy system is inefficient to address the system's uncertainty since it lacks of any automatic learning capabilities. For this reason, further researches, which incorporate the fuzzy logic to adaptive NN control, have been proposed to improve the overall learning performance. In [12], FNN is developed for a rehabilitation exoskeleton robot controller such that the system is able to satisfy the trajectory tracking accuracy under environmental disturbances and parametric uncertainties. By considering the uniform boundedness and state constraint, $\mathrm{He}$ et al. [13] proposed an adaptive FNN control to address the interaction problem of robot.

Nevertheless, a key problem in the aforemention algorithms is that the neural nodes for $\mathrm{NN}$ or the logic rules for fuzzy system are specified in terms of the expert experiences. Once the motion trajectory or working environment is changed, they need to redesign which may result in inefficient training process and poor generation ability. Recently, inspiring by random vector functional-link neural networks (RVFLNN) [15], a novel framework named broad learning system [14] has been proposed and extensively utilized for pattern recognition and classification [16]-[18]. By taking the advantages of RVFLNN, BLS can offer acceptable generalization and expansion performance though increasing the neural nodes dynamically. Herein, this inspires us to bring a forward solution which incorporates BLS and adaptive impedance control to enhance the capability of FNN's generalization.

In this context, we propose a broad fuzzy neural control framework and apply it to a constrained manipulator which interact with the unknown environment. Particularly, the problem of robot-environment interaction is tackled by impedance learning and BFNN is utilized to approximate the unknown dynamic model. Different from the existing researches, the major contributions of this work are that the proposed framework provides new insights into impedance control from incorporating the BLS and FNN to handle the unknown plant model. Further more, state constraint is 
achieved and the closed-loop stability in the process of training is satisfied by using a tan-type Lyapunov function [19].

\section{PROBLEM FUNDAMENTALS}

\section{A. Dynamic and Impedance Model}

In this paper, we discuss the problems of unknown plant model approximation, state constraint and impedance control through investigating the adaptive control and robotenvironment interaction. Since the $n$-link manipulator is controlled in the Cartesian space, we consider the dynamic model based on Lagrange-Euler form as

$$
D_{x}(q) \ddot{x}+C_{x}(q, \dot{q}) \dot{x}+G_{x}(q)=\tau_{x}-\tau_{e}
$$

where $x \in \mathbb{R}^{d}$ is the end-effector's coordinators in Cartesian space; $q \in \mathbb{R}^{d}$ is the joint angle vector; $\tau_{x} \in \mathbb{R}^{d}$ is the control torque regulated by the adaptive controller and $\tau_{e} \in$ $\mathbb{R}^{d}$ denotes the impedance force while interacting with the environment at end-effector; $D_{x}(q) \in \mathbb{R}^{d \times d}$ is a symmetric and positive definite inertia matrix of the manipulator; $C_{x}(q, \dot{q}) \in \mathbb{R}^{d \times d}$ and $G_{x}(q) \in \mathbb{R}^{d}$ represent the Coriolis and centrifugal forces vector and gravity torques respectively which are derived from joint space to Cartesian space as follows:

$$
\begin{aligned}
D_{x}(q)= & J^{-T} D(q) J^{-1} \\
C_{x}(q, \dot{q})= & J^{-T} C(q, \dot{q}) J^{-1} \\
& -J^{-T} M(q) J^{-1} J^{-1} \\
G_{x}(q)= & J^{-T} G(q)
\end{aligned}
$$

where $J \in \mathbb{R}^{d \times d}$ is a non-singular Jacobin matrix of plant.

For the purpose of this work, the manipulator is required to respond to a desirable compliance behavior while contacting with the unknown environment in the presence of model uncertainties. The relationship between the target impedance model and close-loop system can be defined as

$$
D_{m}\left(\ddot{x}_{d}-\ddot{x}\right)+C_{m}\left(\dot{x}_{d}-\dot{x}\right)+G_{m}\left(x_{d}-x\right)=\tau_{e}
$$

where $D_{m}, C_{m}$ and $G_{m}$ denote the inertia, damping and stiffness matrices which can be specified by user beforehand, $x_{d} \in \mathbb{R}^{d}$ denotes the desired reference trajectory. When the manipulator works in free space, which means there is no obstruction during the tracking of end-effector, it can be obtained that $x=x_{d}, \forall t>0$ such that $\tau_{e}=0$.

\section{B. Broad Learning System}

Evolving from random vector functional-link neural networks, BLS has been demonstrated its efficient approximation and generation ability in recognition and classification fields. Compared with the conventional RVFLNN, the innovation of BLS is that a set of feature mappings have been used to replace the input vector of the hidden layer. Also, this framework has a capability of generation by using the enhancement nodes which are generated and then incremented in terms of the feature mappings.

The illustration of the BLS is shown in Fig. 1. In this structure, the input and output variables are denoted as $X$

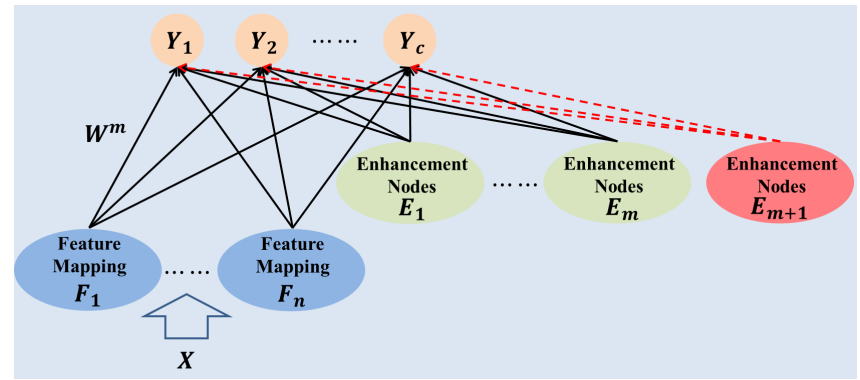

Fig. 1: Schematic diagram of BLS

and $Y \in \mathbb{R}^{N \times C}$ respectively. For the $n$ feature mappings, they can be obtained by considering the input data $X$ as

$$
F_{i}=\psi\left(X W_{f_{i}}+\beta_{f_{i}}\right) \quad i=1 \cdots n
$$

where $W_{f_{i}}$ and $\beta_{f_{i}}$ are generated randomly at the stage of network initialization and then keep unchanged in the process of network training. Then, the enhancement nodes can be denoted as

$$
E_{j}=\xi\left(Z^{n} W_{e_{j}}+\beta_{e_{j}}\right) \quad j=1 \cdots m
$$

where $\psi(\cdot)$ and $\xi(\cdot)$ are the transfer functions. Hence, the network output is represented as

$$
\begin{aligned}
Y & =\left[F_{1}, \cdots, F_{n} \mid \xi\left(F^{n} W_{e_{1}}+\beta_{e_{1}}\right),\right. \\
& \left.\cdots, \xi\left(F^{n} W_{e_{m}}+\beta_{e_{m}}\right)\right] W^{m} \\
& =\left[F_{1}, \cdots, F_{n} \mid E_{1}, \cdots, E_{m}\right] W^{m} \\
& =\left[F^{n} \mid E^{m}\right] W^{m} \\
& =G^{m} W^{m}
\end{aligned}
$$

For the recognition and classification problems, the output vectors are usually given beforehand and the neural weight matrix $W^{m}$ can be yield by the pseudo-inverse of $\left[F^{n} \mid E^{m}\right]$, i.e. $W^{m}=\left[F^{n} \mid E^{m}\right]^{+} Y$. Furthermore, an enhancement node can be inserted into the original network such that a higher accurate approximation can be achieved. In this case, the augmented layer is denoted as

$$
\begin{aligned}
G^{m+1} & =\left[G^{m} \mid E_{m+1}\right] \\
& =\left[G^{m} \mid \xi\left(Z^{n} W_{e_{j+1}}+\beta_{e_{j+1}}\right)\right]
\end{aligned}
$$

Similarly, the incremented weights and biases which are used to derive the $m+1$ enhancement node from mapped features are generated randomly.

\section{Broad Fuzzy Neural Network}

In this part, the principle of the broad fuzzy neural network framework will be described in detail. Previous subsection has shown the approximation capability of the BLS. Fig. 2 illustrates the schematic diagram of the proposed BFNN. There are four layers in this framework. Layer 1 contains the network input vectors $x_{n}$. Then the input variables transmit to layer 2. In this technical note, we apply the Gaussian NN as the transfer membership function, which is defined as the following format from the robotic neural control point of view:

$$
\xi_{i, j}\left(x_{i}\right)=\exp \left[\frac{-\left(x_{i}-c_{i, j}\right)^{T}\left(x_{i}-c_{i, j}\right)}{\eta^{2}}\right]
$$




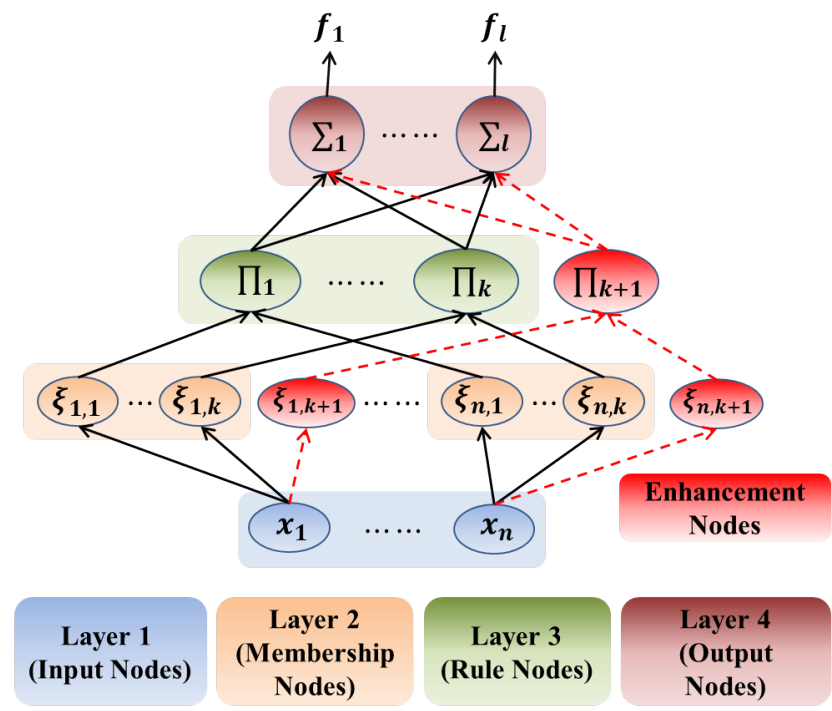

Fig. 2: Schematic diagram of broad fuzzy neural network

where $\xi_{i}=\left[\xi_{i, 1}, \xi_{i, 2}, \ldots, \xi_{i, k}\right]^{T} . c_{i, j}$ and $\eta$ denote the center and bias of the membership function respectively. In this layer, the initial number of membership node is defined as $k=1$ which will be incremented dynamically in terms of the input variables. First, we define the norm distance between the input variables and Gaussian centers as the incremental criterion

$$
d\left(x, c_{i}\right)=\left\|x-c_{i}\right\|
$$

The membership nodes will be augmented to $\xi_{i, k+1}$ while the distance $d\left(x, c_{o}\right)$ is larger than the predefined threshold $\Theta$, where $c_{o}$ represents the nearest center from the input vector. The enhancement node is defined as a new tuple

$$
\xi_{i, k+1}=<c_{i, k+1}, \eta>
$$

where $c_{i, k+1}=\beta\left\|x_{i}-\bar{c}\right\|$. Here, $\bar{c}=\frac{\sum_{i=1}^{l} c_{i}}{k}$ denotes an average position related to the $l$ th nearest centers $c_{i}$.

Next, the rule nodes in layer 3 denote the fuzzy rules which are calculated by $m$-norm product operation as follow:

$$
s_{j}=\prod_{i=1}^{n} \xi_{i, j}\left(x_{i}\right)
$$

Hence, the continuous function approximated by BFNN can be represented as

$$
\begin{aligned}
f(x) & =W^{* T} \vartheta(x)+\varepsilon \\
& = \begin{cases}\frac{\sum_{j=1}^{k} w_{j} s_{j}}{\sum_{j=1}^{k} s_{j}}+\varepsilon & d(x, \bar{c}) \geqslant \Theta \\
\frac{\sum_{j=1}^{k+1} w_{j} s_{j}}{\sum_{j=1}^{k+1} s_{j}}+\varepsilon & \text { otherwise }\end{cases}
\end{aligned}
$$

where $W^{* T}$ is the desired broad fuzzy $\mathrm{NN}$ weight vector and $\left\|\varepsilon_{i}\right\|<\varepsilon_{i}^{*}$ denotes the approximation error.

\section{Adaptive Bfnn Impedance Controller Design}

In this section, the design of adaptive BFNN control scheme by means of impedance learning under the unknown environment is elaborated. Fig. 3 shows the configuration of the proposed controller.

In general, we first define the Cartesian tracing error signals as

$$
\begin{aligned}
& z_{1}=x-x_{d} \\
& z_{2}=\dot{x}-\alpha_{1}
\end{aligned}
$$

where $x_{d} \subset \Omega_{d}$ is the reference trajectory, $\alpha_{1}$ is the virtual controller which will be discussed hereinafter. For these condition, the dynamic model (1) can be rewritten as the state function

$$
\begin{aligned}
& \dot{x}_{1}=x_{2} \\
& \dot{x}_{2}=D_{x}^{-1}\left[\tau_{x}-\tau_{e}-C_{x}(q, \dot{q}) x_{2}-G_{x}(q)\right]
\end{aligned}
$$

where $x_{1}=x$ and $x_{2}=\dot{x}$. In practical term, robotic manipulator often works under many restrictions including the constraints of position and velocity. For these reasons, a Barrier Lyapunov Function, which is defined as follows [18], is adopted to code with the state constraint problem in this paper:

$$
V=\frac{\mu^{2}}{\pi} \tan \left(\frac{\pi \chi^{2}}{2 \mu^{2}}\right)
$$

where $\chi$ is the system state which is aimed to satisfy the constraint with $\|\chi\|<\mu$. According to the L'Hospital's rule, it can be concluded that (18) can be replaced by the quadratic form as follow if the constraint is omitted:

$$
\lim _{\mu \rightarrow \infty} \frac{\mu^{2}}{\pi} \tan \left(\frac{\pi \chi^{2}}{2 \mu^{2}}\right)=\frac{1}{2} \chi^{2}
$$

Particularly, the Cartesian tracking error $z_{1}$ is constrained in a specific area through the tan-type Lyapunov function, i.e. $\left|z_{1 i}\right|<\mu_{i},(i=1,2, \cdots, n)$. Thus, we can specify the virtual control $\alpha_{1}$ in (15) as

$$
\alpha_{1}=-K_{1} \Phi+\dot{x}_{d}
$$

where $\Phi=\left[\phi_{1}, \phi_{2}, \cdots, \phi_{n}\right]^{T}$ is a nonsingularity matrix in terms of L'Hospital's rule with $\phi_{n}=\frac{\mu_{n}^{2}}{2 \pi z_{1 n}} \sin \left(\frac{\pi z_{1 n}^{2}}{\mu_{n}^{2}}\right), K_{1}=$ $\operatorname{diag}\left[k_{11}, \cdots, k_{1 n}\right]$ is a positive constant vector.

Note that the proposed scheme is used to contact with the unknown environment. By taking the contact force $\tau_{e}$ into account, the adaptive control torque is designed as

$$
\tau_{x}=-\rho+\hat{D}_{x} \dot{\alpha}_{1}+\hat{C}_{x} \alpha_{1}+\hat{G}_{x}-K_{p} z_{2}-K_{r} \operatorname{sgn}\left(z_{2}\right)+\tau_{e}
$$

where $\rho=\left[\rho_{1}, \cdots, \rho_{n}\right]^{T}$ with $\rho_{n}=z_{1 n} / \cos ^{2}\left(\frac{\pi z_{1 n}^{2}}{2 \mu_{n}^{2}}\right), \hat{D}, \hat{C}$ and $\hat{G}$ are the estimates of robotic dynamic models which is approximated by BFNN (14) and

$$
\begin{aligned}
D_{x} & =W_{D}^{* T} \vartheta_{D}+\varepsilon_{D} \\
C_{x} & =W_{C}^{* T} \vartheta_{C}+\varepsilon_{C} \\
G_{x} & =W_{G}^{* T} \vartheta_{G}+\varepsilon_{G}
\end{aligned}
$$

Here, we assume that $(\tilde{\cdot})=(\hat{\cdot})-(\cdot)^{*}$. The error dynamic is calculated as follow by substituting the control torque (21) into the robotic model (1):

$$
\begin{aligned}
D_{x} \dot{z}_{2} & =-\rho-C_{x} z_{2}+\tilde{D}_{x} \dot{\alpha}_{1}+\tilde{C}_{x} \alpha_{1}+\tilde{G}_{x} \\
& -K_{p} z_{2}-K_{r} \operatorname{sgn}\left(z_{2}\right)+E_{x}
\end{aligned}
$$




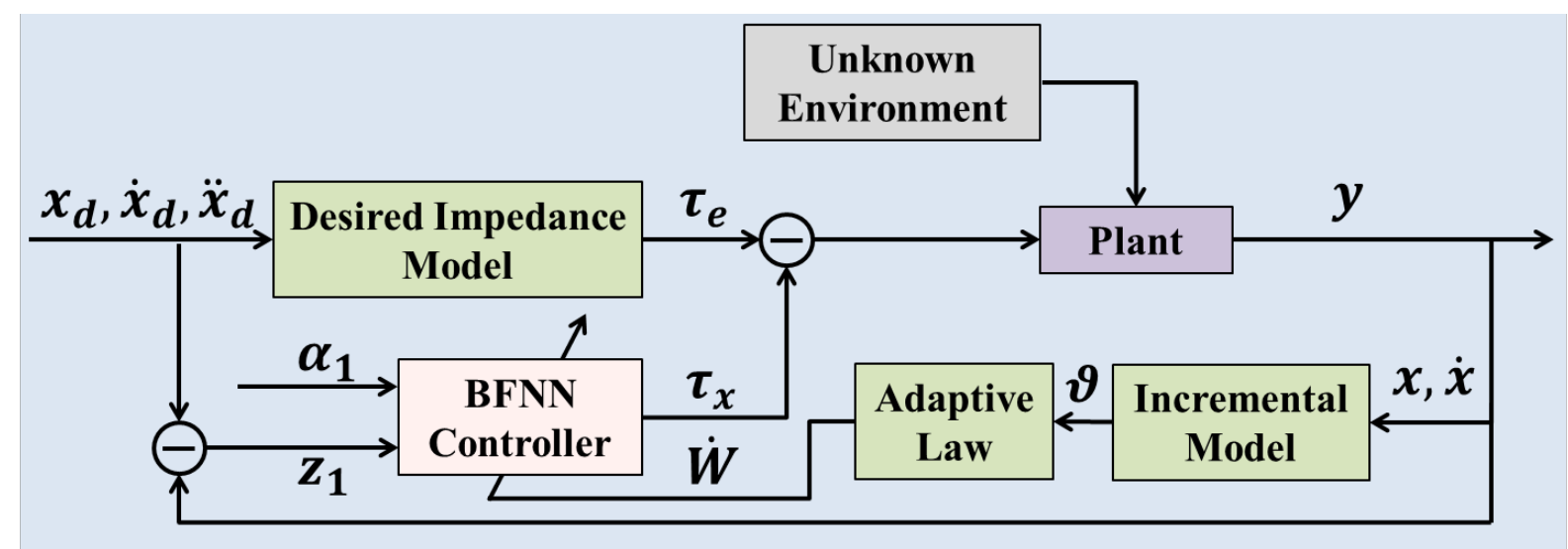

Fig. 3: Control diagram of the BFNN controller

where $E_{x}=\varepsilon_{D} \dot{\alpha}_{1}+\varepsilon_{C} \alpha_{1}+\varepsilon_{G}$ with $K_{r}>\left\|E_{x}\right\|$.

Next, the Lyapunov approach is used to design a stable controller and adaptive updated law of BFNN (14) by considering the following Lyapunov function:

$$
\begin{aligned}
V_{1}(t) & =\sum_{i=0}^{n} \frac{\mu_{i}^{2}}{\pi} \tan \left(\frac{\pi z_{1 i}^{2}}{2 \mu_{i}^{2}}\right)+\frac{1}{2} z_{2}^{T} D_{x} z_{2} \\
& +\frac{1}{2} \tilde{W}_{D}^{T} \tilde{W}_{D}+\frac{1}{2} \tilde{W}_{C}^{T} \tilde{W}_{C}+\frac{1}{2} \tilde{W}_{G}^{T} \tilde{W}_{G}
\end{aligned}
$$

Since $\left[\dot{D}_{x}-2 C_{x}\right]$ is a skew-symmetric matrix, we take the derivative of the candidate function by means of the error dynamic (23) which result in

$$
\begin{aligned}
\dot{V}_{1}(t) & =-\sum_{i=0}^{n} \frac{k_{1 i} \mu_{i}^{2}}{\pi} \tan \left(\frac{\pi z_{1 i}^{2}}{2 \mu_{i}^{2}}\right)-z_{2}^{T} K_{p} z_{2} \\
& +z_{2}^{T}\left(E_{x}-K_{r} \operatorname{sgn}\left(z_{2}\right)\right) \\
& +z_{2}^{T}\left(\tilde{W}_{D}^{T} \vartheta_{D} \dot{\alpha}_{1}+\tilde{W}_{C}^{T} \vartheta_{C} \alpha_{1}+\tilde{W}_{G}^{T} \vartheta_{G}\right) \\
& +\tilde{W}_{D}^{T} \dot{\hat{W}}_{D}+\tilde{W}_{C}^{T} \hat{\hat{W}}_{C}+\tilde{W}_{G}^{T} \dot{\hat{W}}_{G}
\end{aligned}
$$

The adaptive updated laws are designed as

$$
\begin{aligned}
& \dot{\hat{W}}_{D}=-\vartheta_{D} \dot{\alpha}_{1} z_{2}-\delta_{D} \hat{W}_{D} \\
& \dot{\hat{W}}_{C}=-\vartheta_{C} \alpha_{1} z_{2}-\delta_{C} \hat{W}_{C} \\
& \dot{\hat{W}}_{G}=-\vartheta_{G} z_{2}-\delta_{G} \hat{W}_{G}
\end{aligned}
$$

where $\delta_{D}, \delta_{C}, \delta_{G}$ are the robust items for the BFNN impedance controller. Substituting the update laws (26) into (25), the differential Lyapunov function (25) can be rewriten as

$$
\begin{aligned}
\dot{V}_{1}(t) & =-\sum_{i=0}^{n} \frac{k_{1 i} \mu_{i}^{2}}{\pi} \tan \left(\frac{\pi z_{1 i}^{2}}{2 \mu_{i}^{2}}\right)-z_{2}^{T} K_{p} z_{2} \\
& -\delta_{D} \tilde{W}_{D}^{T} \hat{W}_{D}-\delta_{C} \tilde{W}_{C}^{T} \hat{W}_{C}-\delta_{G} \tilde{W}_{G}^{T} \hat{W}_{G}
\end{aligned}
$$

Taking the equality $-\tilde{W}^{T} \hat{W}=-\tilde{W}^{T}\left(W^{*}+\tilde{W}\right)$ and the Young's inequality $-\tilde{W}^{T} W^{*} \leqslant \frac{1}{2}\left(\tilde{W}^{T} \tilde{W}+W^{* T} W^{*}\right)$ into ac- count, we can get

$$
\begin{aligned}
\dot{V}_{1}(t) & \leqslant-\sum_{i=0}^{n} \frac{k_{1 i} \mu_{i}^{2}}{\pi} \tan \left(\frac{\pi z_{i}^{2}}{2 \mu_{i}^{2}}\right)-z_{2}^{T} K_{p} z_{2} \\
& -\frac{1}{2}\left(\tilde{W}_{D}^{T} \tilde{W}_{D}+\tilde{W}_{C}^{T} \tilde{W}_{C}+\tilde{W}_{G}^{T} \tilde{W}_{G}\right) \\
& +\frac{1}{2}\left(W_{D}^{* T} W_{D}^{*}+W_{C}^{* T} W_{C}^{*}+W_{G}^{* T} W_{G}^{*}\right) \\
& \leqslant-\gamma V_{1}(t)+v
\end{aligned}
$$

where $\gamma=\min \left(2 k_{11}, 2 k_{12}, \cdots, 2 k_{1 n}, \frac{2 \lambda_{\min }\left(K_{p}\right)}{\lambda_{\max }\left(D_{x}\right)}\right)$ with $\lambda$ respects the eigenvalue of $(\cdot), v=\frac{1}{2}\left(W_{D}^{* T} W_{D}^{*}+W_{C}^{* T} W_{C}^{*}+W_{G}^{* T} W_{G}^{*}\right)$. Hence, the control parameters should be specified as $\left|K_{p}\right|>0$ and $\left|K_{1}\right|>0$ such that the differential Lyapunov candidate is negative definite. Multiplying (28) by $e^{\gamma t}$, we have

$$
\frac{d\left(V_{1}(t) e^{\gamma t}\right)}{d t} \leqslant v e^{\gamma t}
$$

Computing the integral of (29) and yields

$$
\begin{aligned}
V_{1}(t) & \leqslant e^{-\gamma t} V_{1}(0)+\frac{v}{\gamma}\left(1-e^{-\gamma t}\right) \\
& \leqslant V_{1}(0)+\frac{v}{\gamma}
\end{aligned}
$$

Considering $z_{1}$, we can get

$$
\frac{1}{2} z_{1}^{T} z_{1} \leqslant V_{1}(0)+\frac{v}{\gamma}
$$

In other words, $\left\|z_{1}\right\| \leqslant 2\left(V_{1}(0)+\frac{v}{\gamma}\right)$. It can be concluded from (29)- (31) that the dynamics system (17) and control signal (21) are bounded. The tracking error $z_{1}$ is bounded and converges to the specific compact set $\Omega_{z_{1}}$ in terms of (31). Once choosing the reasonable design parameters, it is able to further shown that the target impedance can be achieved and the tracking errors are uniformly ultimately bounded (UUB). In this way, the proposed controller can enable the control system stable in sense of Lyapunov.

\section{SIMULATION STUDIES}

In this section, a comparative simulation both in interaction case and free movement case to verify the effectiveness of the proposed algorithm is conducted. Without loss of 
TABLE I: Parameters of the manipulator

\begin{tabular}{cccc}
\hline Parameter & Description & Value & Unit \\
\hline$m_{1}$ & Mass of link 1 & 10.0 & $\mathrm{~kg}$ \\
$m_{2}$ & Mass of link 2 & 10.0 & $\mathrm{~kg}$ \\
$l_{1}$ & Length of link 1 & 1.0 & $\mathrm{~m}$ \\
$l_{2}$ & Length of link 2 & 1.0 & $\mathrm{~m}$ \\
$I_{1}$ & Inertia of link 1 & 0.83 & $\mathrm{kgm}^{2}$ \\
$I_{2}$ & Inertia of link 2 & 0.83 & $\mathrm{kgm}^{2}$ \\
$g$ & Gravity acceleration & 9.8 & $\mathrm{~m} / \mathrm{s}^{2}$ \\
\hline
\end{tabular}

generality, the end-effector a two-DOF manipulator equipped with a force sensor to detect the disturbance force is investigated to move in the free space and then interacted with a fixed wall as shown in Fig. 4. In this scenario, the wall is simply treated as a rigid object which can be modelled as

$$
f_{w}=\left[\begin{array}{l}
f_{w x} \\
f_{w y}
\end{array}\right]=\left[\begin{array}{c}
k_{w}\left(x(1)-x_{w}\right) \\
0
\end{array}\right]
$$

where $k_{w}$ denotes rigid of the wall, $x_{w}$ is wall's coordinate at x-axis. Simply, we assume that there is no friction disturbance while the manipulator is sliding along y-axis at the wall such that $f_{w y}=0$.

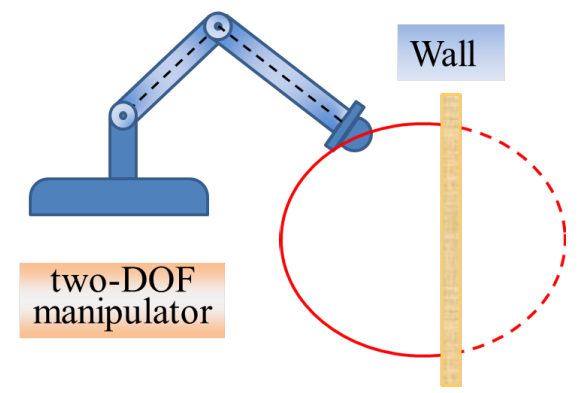

Fig. 4: Simulation scenario for two-DOF manipulator

As the system has no information about the wall and dynamic of manipulator, the purpose of designing such scenario is to examine the proposed controller's capability for tackling the tracking problem both in free space and in constraint space, i.e. contacting with the unknown wall.The format of Lagrange-Euler dynamic matrices $D_{x}, C_{x}$ and $G_{x}$ in (1) can be found in [20]. Specifically, the model parameters is specified as Table I.

In this test, the desired trajectory is design as

$$
x_{d}(t)=\left[\begin{array}{c}
1-0.3 \cos (t) \\
1+0.3 \sin (t)
\end{array}\right]
$$

which denotes a circle located at $[1,1]$ with $0.3 \mathrm{~m}$ radius. The initial position of the manipulator is specified as $[0.58 \pi,-0.58 \pi]$ in joint space and the wall is located at $x_{w}=1.1 \mathrm{~m}$. In this simulation, the target impedance model is designed as $D_{m}=\operatorname{diag}[0.1], C_{m}=\operatorname{diag}[20]$ and $G_{m}=\operatorname{diag}[100]$. The adaptive impedance control parameters are specified as $K_{1}=\operatorname{diag}[40,60], K_{r}=\operatorname{diag}[20,20], K_{p}=$ $\operatorname{diag}[80,80]$ and $\mu=[0.5,0.5]^{T}$. Furthermore, the BFNN updated law is specified as (26) with $\delta_{D}=1.5, \delta_{C}=1.5$ and

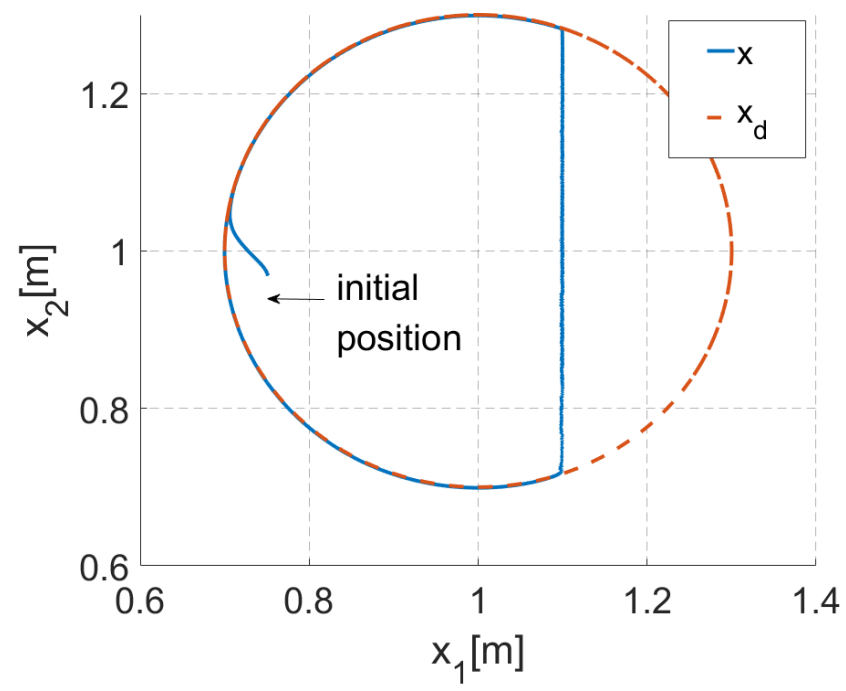

Fig. 5: Simulation of trajectory in Cartesian space

$\delta_{G}=2.0$. The distance threshold $\Theta$ for incremental nodes is chosen as $\Theta_{D}=0.3, \Theta_{C}=0.5$ and $\Theta_{G}=0.2$ respectively.

In order to verify the effectiveness of the proposed framework, the manipulator is required to move in free space and then slide along the unknown wall which will produce a contact force while contacting. Fig. 5 shows the result of tracking simulation. It shows that the adaptive BFNN impedance controller can provide a smooth tracking performance and has an ability to tackle the interaction problem while the environment is changed.

Fig. 6 gives the comparison of tracking performance and control torque signal. From Fig. 6(a), the manipulator is able to track the desired trajectory in free space perfectly. However, there are a few oscillations occurring due to the change of environment while the end-effector is starting to contact with the wall. However, fast convergence can be achieved towards the proposed control.

In addition, the number of membership nodes for $\hat{D}_{x}$, $\hat{C}_{x}$ and $\hat{G}_{x}$ approximation which are incremented adaptively during the training phase is shown in Fig. 7. Instead of specifying the parameters of neuron nodes beforehand which mainly depend on the expert experience and tend to be inconvenient, the proposed network has an ability to expand automatically in terms of the state variables. At the same time, the growing number of incremental nodes can be limited by the distance threshold $\Theta$.

\section{CONCLusion}

In this paper, we have proposed an adaptive broad fuzzy $\mathrm{NN}$ control to improve the performance of robot-environment interaction by adopting the impedance learning. The enhancement membership nodes are expanded dynamically by considering the input variables and the uncertainty of robotic dynamic model is addressed by using the proposed BFNN framework. The stability and convergence of the closedloop system has been proven rigorously by the Lyapunov 

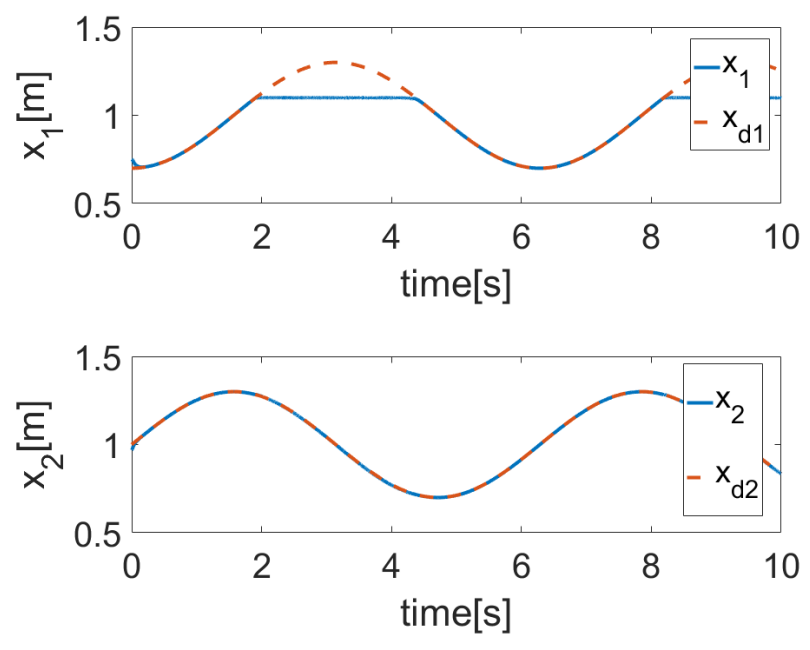

(a) Position tracking
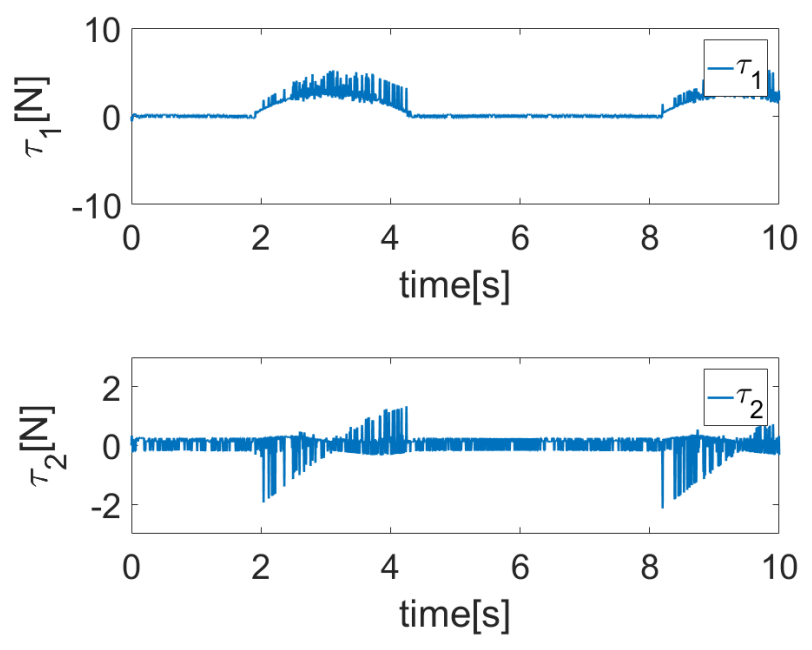

(b) Control torque

Fig. 6: Control performance with BFNN

Function. We demonstrate from the simulation results that the proposed methodology is valid for the adaptive impedance control system and it can also provide the favourable tracking performance.

\section{REFERENCES}

[1] M. H. Raibert and J. J. Craig, Hybrid position/force control of manipulators, Asme J of Dynamic Systems Measurement and Control, vol. 102, no. 2, pp. 126133, 1981.

[2] N. Hogan, Impedance control: An approach to manipulation, in American Control Conference, 2009.

[3] Yang C, Chao Z, Yang C, et al. A Learning Framework of Adaptive Manipulative Skills from Human to Robot, IEEE Transactions on Industrial Informatics, vol. PP, no. 99, pp. 1-1, 2018.

[4] C. C. Cheah and D. Wang, Learning impedance control for robotic manipulator, IEEE Trans Robotics and Automation, vol. 14, no. 3, pp. 452465, 1998.

[5] C. Yang and E. Burdet, A model of reference trajectory adaptation for interaction with objects of arbitrary shape and impedance, in Ieee/rsj International Conference on Intelligent Robots and Systems, 2011, pp. 41214126.

[6] Y. Li and S. S. Ge, Impedance learning for robots interacting with unknown environments, IEEE Transactions on Control Systems Technology, vol. 22, no. 4, pp. 14221432, 2014.

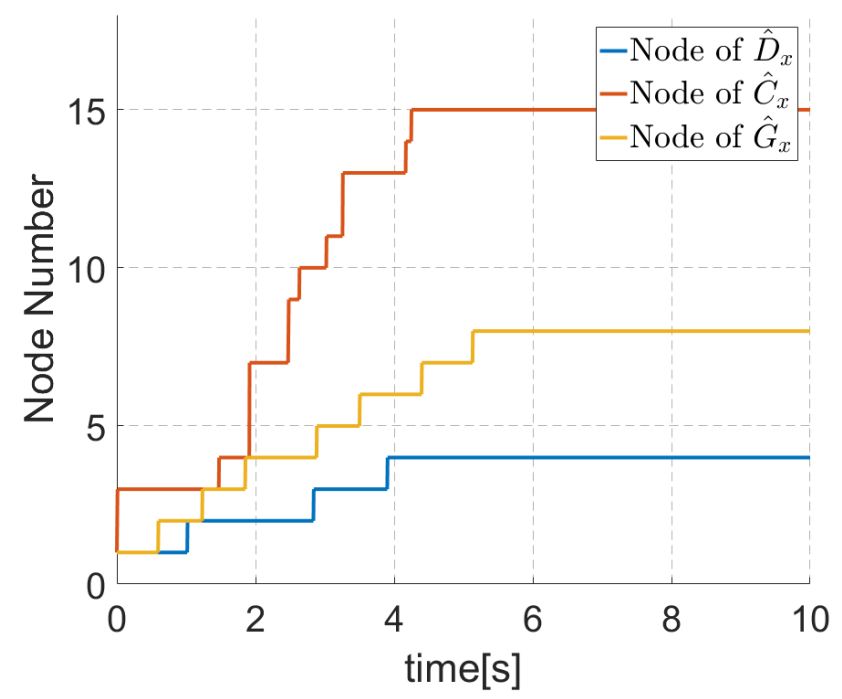

Fig. 7: The number of membership nodes in BFNN

[7] H. Li, L. Bai, L. Wang, Q. Zhou, and H. Wang, Adaptive neural control of uncertain nonstrict-feedback stochastic nonlinear systems with output constraint and unknown dead zone, IEEE Transactions on Systems Man and Cybernetics Systems, vol. 47, no. 8, pp. 20482059, 2017.

[8] S. L. Dai, C. Wang, and M. Wang, Dynamic learning from adaptive neural network control of a class of nonaffine nonlinear systems, IEEE Transactions on Neural Networks and Learning Systems, vol. 25, no. 1, pp. 111123, 2013.

[9] H. Modares, F. L. Lewis, and M. B. Naghibi-Sistani, Adaptive optimal control of unknown constrained-input systems using policy iteration and neural networks, IEEE Transactions on Neural Networks and Learning Systems, vol. 24, no. 10, pp. 15131525, 2013.

[10] S. S. Ge, C. C. Hang, L. C. Woon, and X. Q. Chen, Impedance control of robot manipulators using adaptive neutral network. Int.j.intell.control Syst, no. 3, pp. 433452, 1998.

[11] L. Ping, S. S. Ge, and W. Chen, Impedance control for human-robot interaction with an adaptive fuzzy approach, in Control and Decision Conference, 2017.

[12] Q. Wu, X. Wang, C. Bai, and H. Wu, Development of an rbfn-based neural-fuzzy adaptive control strategy for an upper limb rehabilitation exoskeleton, Mechatronics, vol. 53, pp. 8594, 2018.

[13] W. He and Y. Dong, Adaptive fuzzy neural network control for a constrained robot using impedance learning. IEEE Transactions on Neural Networks and Learning Systems, vol. 29, no. 4, pp. 11741186 , 2018.

[14] C. Chen and Z. Liu, Broad learning system: An effective and efficient incremental learning system without the need for deep architecture, IEEE Transactions on Neural Networks and Learning Systems, vol. 29, no. 1, pp. 1024, 2017.

[15] Igelnik, B. and Y. H. Pao, Stochastic choice of basis functions in adaptive function approximation and the functional-link net, IEEE Trans Neural Netw, vol. 6, no. 6, pp. 13201329, 1995.

[16] Z. Liu, Z. Jin, and C. L. P. Chen, Broad learning system: Feature extraction based on k-means clustering algorithm, in International Conference on Information, 2017.

[17] F. Shuang and C. L. P. Chen, Fuzzy broad learning system: A novel neuro-fuzzy model for regression and classification, IEEE Transactions on Cybernetics.

[18] M. Han, S. Feng, C. L. P. Chen, M. Xu, and T. Qiu, Structured manifold broad learning system: A manifold perspective for largescale chaotic time series analysis and prediction, IEEE Transactions on Knowledge and Data Engineering, pp. 11.

[19] X. Jin and J. X. Xu, Iterative learning control for output-constrained systems with both parametric and nonparametric uncertainties, Automatica, vol. 49, no. 8, pp. 25082516, 2013.

[20] S. S. Ge, C. C. Hang, and L. C. Woon, Adaptive neural network control of robot manipulators in task space, IEEE Transactions on Industrial Electronics, vol. 44, no. 6, pp. 746752, 2002. 